\title{
28 Research Suare \\ PFC2D Simulation Research on Arched Structure of Salix Granules in Dense Forming
}

\section{Sha Qianyi ( $\nabla 1104598447 @ q q . c o m)$}

Inner Mongolia University of Science and Technology

\section{Li Zhen}

Inner Mongolia University of Science and Technology

\section{Wang Wei}

Inner Mongolia University of Science and Technology

\section{Li Jinda}

Inner Mongolia University of Science and Technology

\section{Original Article}

Keywords: Salix granules, energy, arched structure, meso

Posted Date: October 25th, 2021

DOl: https://doi.org/10.21203/rs.3.rs-970776/v1

License: (c) (i) This work is licensed under a Creative Commons Attribution 4.0 International License. Read Full License 


\section{Abstract}

The influencing factors of biomass molding play an important role in the entire biomass molding research. This study uses the arch structure to study the relationship between the microscopic and macroscopic influencing factors. Using PFC2D software to carry out uniaxial compression simulation, by changing the two influencing factors of compression speed and forming granularity, the influence of arched structure on the compact forming of Salix granules from the meso level. By analyzing the stressstrain relationship and the changes of various types of energy, the influence of the arched structure on the energy during the compression process is reflected. The research results show that the arched structure formed in the compression process will cause the stagnation or even decrease of the stress and bonding energy; the destruction of the arched structure will also cause a sudden increase in the friction energy of the particles. When the compression speeds are $0.075 \mathrm{~mm} \cdot \mathrm{s}-1,0.1 \mathrm{~mm} \cdot \mathrm{s}-1$, and $0.125 \mathrm{~mm} \cdot \mathrm{s}-1$, the number of particles in the arched structure are 55, 60 and 75, respectively, and the boundary energy is transformed into cohesive energy. The efficiencies are $87.2 \%, 73.8 \%$ and $89.9 \%$, respectively. When the molding particle size is $0.1 \mathrm{~mm}$ to $0.25 \mathrm{~mm}, 0.25 \mathrm{~mm}$ to $0.4 \mathrm{~mm}, 0.4 \mathrm{~mm}$ to $0.55 \mathrm{~mm}$, the first sudden change in stress during the compression molding process is $0.746 \mathrm{MPa}, 0.812 \mathrm{MPa}$ and $1.161 \mathrm{MPa}$.

\section{Introduction}

As one of the most promising new energy sources, biomass energy has the characteristics of large reserves, low pollution and renewable energy compared with traditional energy sources such as petroleum and coal. Countries in the world have made considerable progress in the utilization of biomass energy, but the utilization of biomass particles is still at a relatively slow stage.China is a country with a huge amount of biomass resources. Proper use of biomass can effectively alleviate the current energy crisis, and it also conforms to the scientific concept of sustainable development. The development and utilization of biomass energy has become a part of Chinese national energy strategy.At present, most of the research on the forming mechanism of biomass is still on the influence of macroscopic factors (temperature, moisture, lignin content, etc.) on the forming process. There are few studies on the microscopic influencing factors on biomass molding. In this paper, the influence of compression speed and molding particle size on the molding process is further studied by observing the arch structure.

Salix is a kind of sand shrub widely distributed in Inner Mongolia. Because of its repeated growth biological habit, it is a good raw material for the development of biomass fuel.Due to its shortcomings of inconvenient storage and high transportation cost, it is difficult to use. This article will further discuss this situation.

At present, the research on arching of bulk materials mainly focuses on rock and soil mechanics. The physical properties of Salix granules at the meso-level are similar to those of rock and soil particles, so this paper analyzes them with the help of rock and soil mechanics methods and theories.Rumpf believes that bridging or bridging between particles is one of the main bonding methods inside particles. Wu Zishu and others believe that soil particles will spontaneously produce "wedge tightness", and then form a 
particle arching effect within a certain range.Jia Haili et al. pointed out that the particle arching effect is to generate force first and then form an arched structure.In the simulation process, different compression speeds and forming granularities are used. Analyze the influence of the arched structure from the changes in stress-strain and each group of energy to provide a certain theoretical basis for the production of biomass fuels.

In this study, different compression speeds and molding granularities were used in the PFC2D simulation process. Analyze the influence of the arched structure from the changes in stress-strain and each group of energy to provide a certain theoretical basis for the production of biomass fuels.

\section{Simulation Method}

\subsection{Discrete element model and simulation parameters}

In this paper, the spherical particle model is used for simulation. Compared with the sheet model and the needle model, the spherical model can better reflect the formation of the arched structure in the structure. According to the physical and mechanical properties of Salix granules, the simulation parameters are set as shown in Table 1.Refer to the contact properties between the raw material particles and the contact model in PFC2D. The linear parallel bonded contact model (linearpbond) that can transmit force and torque is selected between the particles. The default between the particles and the mold is the linear contact model (linear).According to the actual simulation process, a mold with a length-to-diameter ratio of $5: 1$ is established and the wall is loaded up and down, and the data is recorded every 5 time steps. Use the ball attribute command to create particles, and use the cmat default command to assign values to the contact parameters to generate a model; wait for the particle state to stabilize, and then use different compression speeds to compress.

With the given parameters in the designated calculation area, the model shown in Figure 1 below is generated. The size of the model is $50 \mathrm{~mm} \times 250 \mathrm{~mm}$, and the size of the model is one-tenth of the actual size.This study focuses on the arched structures , and does not consider the impact of the size effect.

Table 1 Setting of model parameter 


\begin{tabular}{|c|c|c|}
\hline parameter & material & Numerical value \\
\hline Relative modulus $\llbracket$ emod $\square$ & particle & $1.0 \mathrm{e} 9$ \\
\hline \multirow[t]{2}{*}{ density/ $\left[\mathrm{kg} \mathrm{m}^{-3}\right]$} & particle & 650 \\
\hline & mould & no \\
\hline porosity & particle & 0.26 \\
\hline Stiffness ratio & particle & 1.5 \\
\hline ¿kratio $\square$ & mould & 2.0 \\
\hline \multirow[t]{2}{*}{ coefficient of static friction } & granules and granules & 0.07 \\
\hline & mold and pellets & 0.05 \\
\hline \multirow[t]{2}{*}{ sliding friction coefficient } & granules and granules & 0.03 \\
\hline & mold and pellets & 0.03 \\
\hline
\end{tabular}

\subsection{Experimental design and theoretical analysis}

Compression speed and molding particle size have a significant impact on the molding characteristics of biomass fuel. Wang Yunshan ${ }^{[4]}$ obtained the least specific energy consumption of corn stover and lignite at a compression speed of $25 \mathrm{~mm} \cdot \mathrm{min}^{-1}$ through experimental analysis.At the same time, the particle size of the material is also one of the important factors affecting fuel formation. Li Zhen et al ${ }^{[5]}$. used the discrete element software EDEM to perform orthogonal analysis on the forming factors of Salix granules, and obtained the best forming characteristics when the particle size is $1 \sim 4 \mathrm{~mm}$, the compression speed is $60 \mathrm{~mm} \cdot \mathrm{min}^{-1}$, and the holding time is $75 \mathrm{~s}$. In this paper, through the influence of compression speed and molding granularity on the arch structure, the macroscopic effect during the molding process is further connected with the microstructure, and the influence of compression speed on fuel molding characteristics is deeply reflected.Since the PFC software cannot directly analyze the influence of temperature and moisture, this paper takes the two influencing factors of compression speed and molding particle size as experimental variables. Since the PFC software sets the contact bonding strength and final molding density in advance, set the same contact bonding strength and final molding density to reflect the compression quality through energy analysis. The arched structure is a good load-bearing structure, but the existence of the arched structure affects the molding quality of Salix pill. Based on the characteristics of the contact network, Sun Qicheng et al ${ }^{[8]}$. revealed the criterion of the size of strong and weak force chains ( $\mathrm{Fc}$ is greater than the average contact force $\langle\mathrm{F}>$ ) and the criterion of angle $\theta \mathrm{c}$ $(\theta c=180 / Z$, where $<Z>$ Is the average coordination number)

During the compression molding process, some particles are in the weak force chain, and the stress is small or even not stressed. In this paper, these particles are considered to be in an arched structure, and the function of the fish language in the PFC2D software is used to realize the criterion of strong and weak force chains, and they are separated. 
Based on the above analysis, this experiment uses the same preset contact bonding model and uses different compression speeds and raw material particle sizes for compression, and selects three sets of data to observe the influence of the arch structure on the molding process for analysis.

2. Analysis of simulation results at different compression speeds

\section{Analysis Of Simulation Results At Different Compression Speeds}

\subsection{Quantity analysis of arched structures}

Simulate with the compression speeds of $0.075 \mathrm{~mm} \cdot \mathrm{s}-1,0.1 \mathrm{~mm} \cdot \mathrm{s}-1$ and $0.125 \mathrm{~mm} \cdot \mathrm{s}-1$, divide them into three groups A, B, and C. Use the polt function in PFC2D to form blocks The number of particles in the arched structure is counted. The total number of particles in the model is 258 . Under the compression speed of $0.075 \mathrm{~mm} \cdot \mathrm{s}-1,0.1 \mathrm{~mm} \cdot \mathrm{s}-1$ and $0.125 \mathrm{~mm} \cdot \mathrm{s}-1$, when the compression produces When the strain is 0.15 , the numbers of particles in the weak force chain are 55,60 , and 75 , respectively.

In the molding process, the faster the compression speed, the more uneven the contact force between the particles, resulting in more arched structures formed between the particles, that is, the more weak force chains formed.The particles are rearranged and appear "wedge-caulking",forming the arched structure to resist vertical forces.

\subsection{Stress-strain analysis}

Under different compression speeds, the stress-strain curve shown in the figure below is obtained. When the strain is 0 to 0.08 , there is almost no change in the stress. The reason is that when the strain is small, the main effect of compression is to reduce the gap between particles and promote contact between particles. When the strain increases, the three curves all show a gradual increase in stress as the strain increases.

It can be seen from the curve that the greater the compression speed, the greater the final stress. In the stress-strain curve, during the compression molding process of the three curves, the stress suddenly decreases. The reason is that the arched structure is destroyed at this stage. By observing the dynamic compression molding process, it can be known that the fluctuation of the stress is caused by the destruction of the arched structure.

It can be known from the stress-strain curve that the faster the compression speed, the greater the final stress on the sample. It is proved that the destruction of the arch structure leads to the reduction of stress during the compression molding process.

\subsection{Energy analysis}

Since the molding process is a quasi-static process, the kinetic energy increases slowly during the entire compression molding process, so the change of the particle kinetic energy is not analyzed. The energy 
obtained by the wall in the compression molding process is called boundary energy, which is equivalent to the total work in mechanical energy; the energy required for bonding between particles is called cohesive energy, which is equivalent to useful work in mechanical energy; friction energy is equivalent to mechanical energy. The useless work. Use the history command to record the boundary energy, cohesive energy and friction energy of the particles during the compression process, and also analyze the conversion rate from boundary energy to cohesive energy.

The various energy change curves in compression molding are shown in Figures 4, 5, and 6 below. In the stage of particle gap reduction during the compression process, the bonding energy between particles remains unchanged, accompanied by a slow increase in friction energy. When an arched structure is formed between the particles, the frictional energy begins to stagnate; the arched structure is destroyed, and the frictional energy begins to increase. The increase in frictional energy during the entire compression molding process is accompanied by the formation and destruction of arched structures.

The conversion efficiency of the boundary energy to the bonding energy at each compression speed is respectively, the efficiency at the compression speed of $0.075 \mathrm{~mm} \cdot \mathrm{s}-1,0.1 \mathrm{~mm} \cdot \mathrm{s}-1$ and $0.125 \mathrm{~mm} \cdot \mathrm{s}-1$ is $87.2 \%, 73.8 \%$ and $89.9 \%$.

\section{Analysis Of Simulation Results Under Different Raw Material Particle Sizes}

\subsection{Arch structure and force chain network analysis}

The force chain network can reflect the macroscopic mechanical properties of the entire particle system through its dynamic response ${ }^{[9,12,14]}$. PFC is used to simulate and observe the arch structure and force chain network formed during the molding process, reflecting the influence of different raw material particle sizes on it.

Select three raw material particle sizes, namely $0.1 \mathrm{~mm}$ to $0.25 \mathrm{~mm}, 0.25 \mathrm{~mm}$ to $0.4 \mathrm{~mm}, 0.4 \mathrm{~mm}$ to $0.55 \mathrm{~mm}$, and divide them into $\mathrm{D}, \mathrm{E}$, and $\mathrm{F}$ groups for compression simulation. The force chain network of the model after the simulation reaches equilibrium is shown in the figure below. The image distinguishes the size of the force chain by the color of the force chain.

From the extension direction of the power chain, it can be judged that the direction of the principal stress is the vertical direction, and the strength of the power chain at the bottom is greater. The average contact force under each particle size is similar, but the larger the particle size of the raw material, the worse the uniformity of the internal contact force, that is, the larger the particle size of the raw material, the greater the influence of the arched structure; the arched structure surrounded by the compression molding process The larger the area.

\subsection{Stress-strain analysis}


In the compression molding process of different raw material particle sizes, the measured stress and strain of each group are shown in Figure 8 below. Due to the different particle sizes of the raw materials of each group, the strain required to achieve close contact inside the particles of each group is also different, that is, the smaller the particle size of the raw material, the greater the strain required to make the particles come into close contact.

\subsection{Stress-strain analysis}

In the compression molding process of different raw material particle sizes, the measured stress and strain of each group are shown in Figure 8 below. Due to the different particle sizes of the raw materials of each group, the strain required to achieve close contact inside the particles of each group is also different, that is, the smaller the particle size of the raw material, the greater the strain required to make the particles come into close contact.

It can be seen from the stress-strain image that as the particle size of the raw material increases, the maximum stress during compression molding also decreases, but the amount of mold compression required for the raw materials of each particle size from close contact with the particles to compression molding is almost the same. When the raw material particle size is $0.1 \mathrm{~mm}$ to $0.25 \mathrm{~mm}, 0.25 \mathrm{~mm}$ to $0.4 \mathrm{~mm}$, $0.4 \mathrm{~mm}$ to $0.55 \mathrm{~mm}$, the stress mutations caused by the destruction of the first arch structure during the molding process are $0.746 \mathrm{MPa}, 0.812 \mathrm{MPa}$ and $1.161 \mathrm{MPa}$. It can be seen that the larger the particle size of the raw material, the more obvious the sudden change in strain, that is, the larger the particle size of the raw material, the more obvious the influence of each arch structure; the smaller the particle size of the raw material, the more arch structures are formed, but The less affected by each arch structure.

\subsection{Energy analysis}

The energy change during compression molding under different molding particle diameters is shown in the figure. The change trend of the cohesive energy of different raw material particle sizes is the same as the trend of its stress-strain curve.

It can be seen from the friction energy curve that the increase in friction energy is not continuous, but in stages. At the beginning of the particle gap reduction stage, the friction energy begins to increase in the same trend, until the particles are in close contact with each other to form an arched structure, and the friction energy stops growing until the arched structure is destroyed, and the friction energy begins to increase. From the point of view of friction energy, the larger the particle size of the raw material, the less friction energy will eventually be generated, and the greater the mutation of friction energy, that is, the greater the mutation of friction energy.

\section{Conclusion}

1) Compare the number of particles in the arched structure at different compression speeds. At compression speeds of $0.075 \mathrm{~mm} \cdot \mathrm{s}-1,0.1 \mathrm{~mm} \cdot \mathrm{s}-1$, and $0.125 \mathrm{~mm} \cdot \mathrm{s}-1$, the number of particles in the arched 
structure are 55,60 , and 75 , respectively.

2) Through the energy curve at different forming speeds, when the arched structure is formed, it will prevent the material gap from further forming, and the frictional energy will enter the stagnation stage until the arched structure is damaged, and the frictional energy will begin to increase. The conversion efficiency of boundary energy to cohesive energy at each compression speed is $87.2 \%, 73.8 \%$, and $89.9 \%$, respectively.

3) Due to the difference of the forming particle size, the strain required to make the particles reach close contact is also different. From the stress-strain curves of different forming particle sizes, it can be seen that the larger the particle size of the raw material, the smaller the number of arched structures formed, the worse the uniformity of contact force, and the greater the range of influence of the arched structure.

4) According to the various energy curves under different raw material particle sizes, for friction energy, the larger the raw material particle size, the less the number of arched structures formed, and the greater the increase in friction energy when the arched structure is broken.

\section{Declaration}

\section{Funding}

Project supported by the National Natural Science Foundation of China (Grant No.51666016) and the Natural Science Foundation of Inner Mongolia Autonomous Region,China(Grant No.2020LH05020).

\section{Acknowledgments}

The authors acknowledge the helpful discussions with LI Zhen and WANG Wei.

\section{Availability of data and material}

Some or all data, models, or code generated or used during the study are available from the corresponding author by request.

\section{Disclosures}

No conflict of interest exists in the submission of this manuscript. The manuscript is approved by all authors for publication. I would like to declare on behalf of my co-authors that the work described is original research that has not been published previously and is not under consideration for publication elsewhere, in whole or in part.

\section{References}

[1]XU Xiao-min, LING Dao-sheng, CHEN Yun-min, HUANG Bo.Correlation of microscopic and macroscopic elastic constants of granular materials based on linear contact model. Chinese Journal of Geotechnical 
Engineering.2010,32(07):991-998.

[2]R. Trueman and R. Castro and A. Halim. Study of multiple draw-zone interaction in block caving mines by means of a large 3D physical model. International Journal of Rock Mechanics and Mining Sciences, 2007, 45(7) : 1044-1051.

[3]X.X. Kong et al. A method to estimate the pressure arch formation above underground excavation in rock mass. Tunnelling and Underground Space Technology incorporating Trenchless Technology Research, 2018, $71:$ 382-390.

[4]WANG Yunshan.Experimental study on Cold Compression Molding Characteristics of Straw and Coal Mixture.Inner Mongolia Agricultural University,2020

[5]Li Zhen,Wang Hongqiang,Gao Yuhang,Yan ,Li,Wang Peng.Discrete element simulation on dense forming characteristics for Salix biomass particles. Forging \& Stamping Technology,2020,45(03):152-158.

[6]Alexis Sagastume Gutiérrez et al. The energy potential of agriculture, agroindustrial, livestock, and slaughterhouse biomass wastes through direct combustion and anaerobic digestion. The case of Colombia. Journal of Cleaner Production, 2020, 269

[7]SUN Q C,WANG G Q.Introduction to the mechanics of particulate matter冈Beijing:China Science Publishing $\square$ Media Ltd $\llbracket 2009: 94+117$ (in Chinese) $\Downarrow$

[8]Sun Qi-Cheng,Jin Feng,Wang Guang-Qian,Zhang Guo-Hua.Force chains in a uniaxially compressed static granular matter in 2D.Acta Physica Sinica,2010,59(01):30-37.

[9]SUN Qi-cheng, XIN Hai-li, LIU Jian-guo, JIN Feng.Skeleton and force chain network in static granular material.Rock and Soil Mechanics,2009,30(S1):83-87.

[10]Guizhen He et al. Comparing centralized and decentralized bio-energy systems in rural China[J].Energy Policy,2013,63:34-43.

[11]YANG Sheng,LI Xiao-qing.Shear simulation and macro meso analysis of sand based on PFC $^{3 D}$.Chinese Journal of Computational Mechanics,2019,36(06):777-783

[12]Li Zhen,Gao Yuhang,Liu Peng,et al.DISCRETE ELEMENT STUDY ON EVOLUTION OF FORCE-CHAIN DURING SALIX GRAINS DENSE MOLDING.Acta Energiae Solaris Sinica,2019,40(11):3186-3195

[13]Cao Zhi,Simulating method by PFC on mechanical propertiesof sand-mudstone and its particle mixture.Chongqing Jiaotong University,2016.

[14]Kingsley Harrop\&hyphen and Williams. Arch in Soil Arching. Journal of Geotechnical Engineering, $1989,115(3):$ : 415-419. 
[15]JIA Hai-li,WANG Cheng-hua,LI Jiang-hong.Discussion on Some Issues in Theory of Soil Arch.Journal of Southwest Jiaotong University,2003(04):398-402.

[16]XIANG Xian-chao,ZHANG Hua,JIANG Guo-sheng,TU Peng-fei.Soil arching effect of anti-slide piles based on particle flow method. Chinese Journal of Geotechnical Engineering,2011,33(03):386-391.

[17]MA Shi-cheng,HU Jun-xia,MA Yi-yue,DONG Hui.Research on mesoscopic-macroscopic mechanical parameters correlation of accumulation of gravel soil based on three dimensional discrete element.Chinese Journal of Computational Mechanics,2016,33(01):73-82.

[18]Wu Zishu,Zhang Limin,Hu Ding.Studies on the Mechanism of Arching Action in Loess.Advanced Engineering Sciences $\ 1995(02): 15-19$.

[19]Kaliyan Nalladurai and Morey R Vance. Natural binders and solid bridge type binding mechanisms in briquettes and pellets made from corn stover and switchgrass. Bioresource technology, 2010, 101(3) : 1082-90.

\section{Figures}




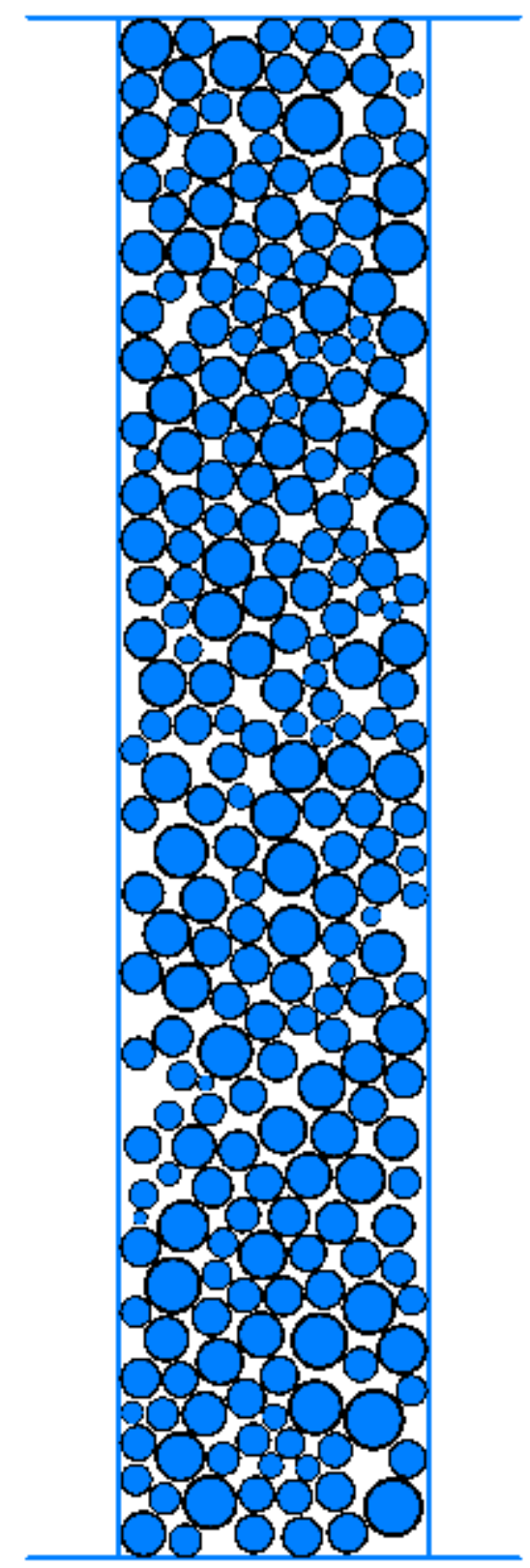

Figure 1

\section{Particle mode}



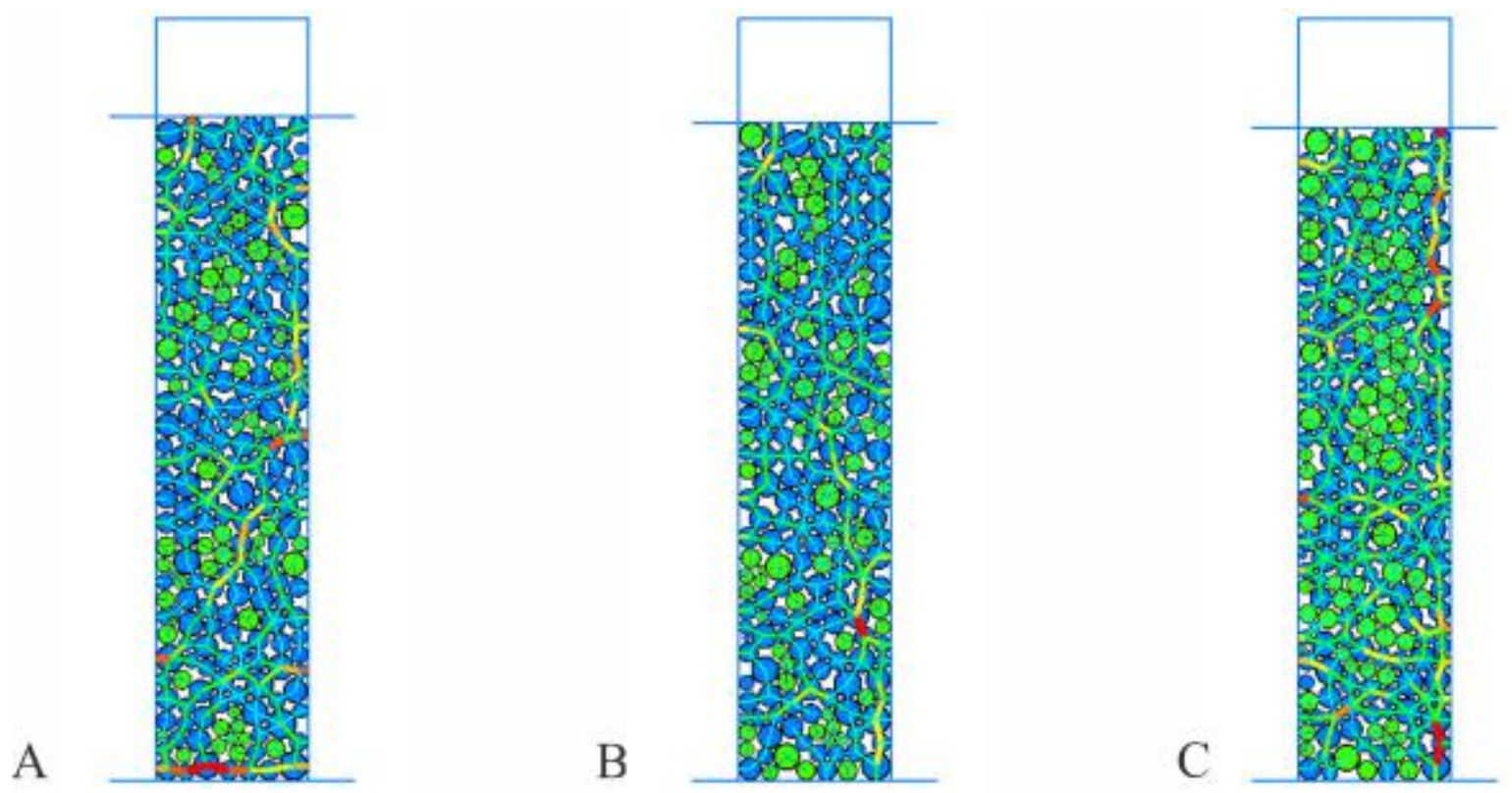

Figure 2

Force chain network under different compression speeds

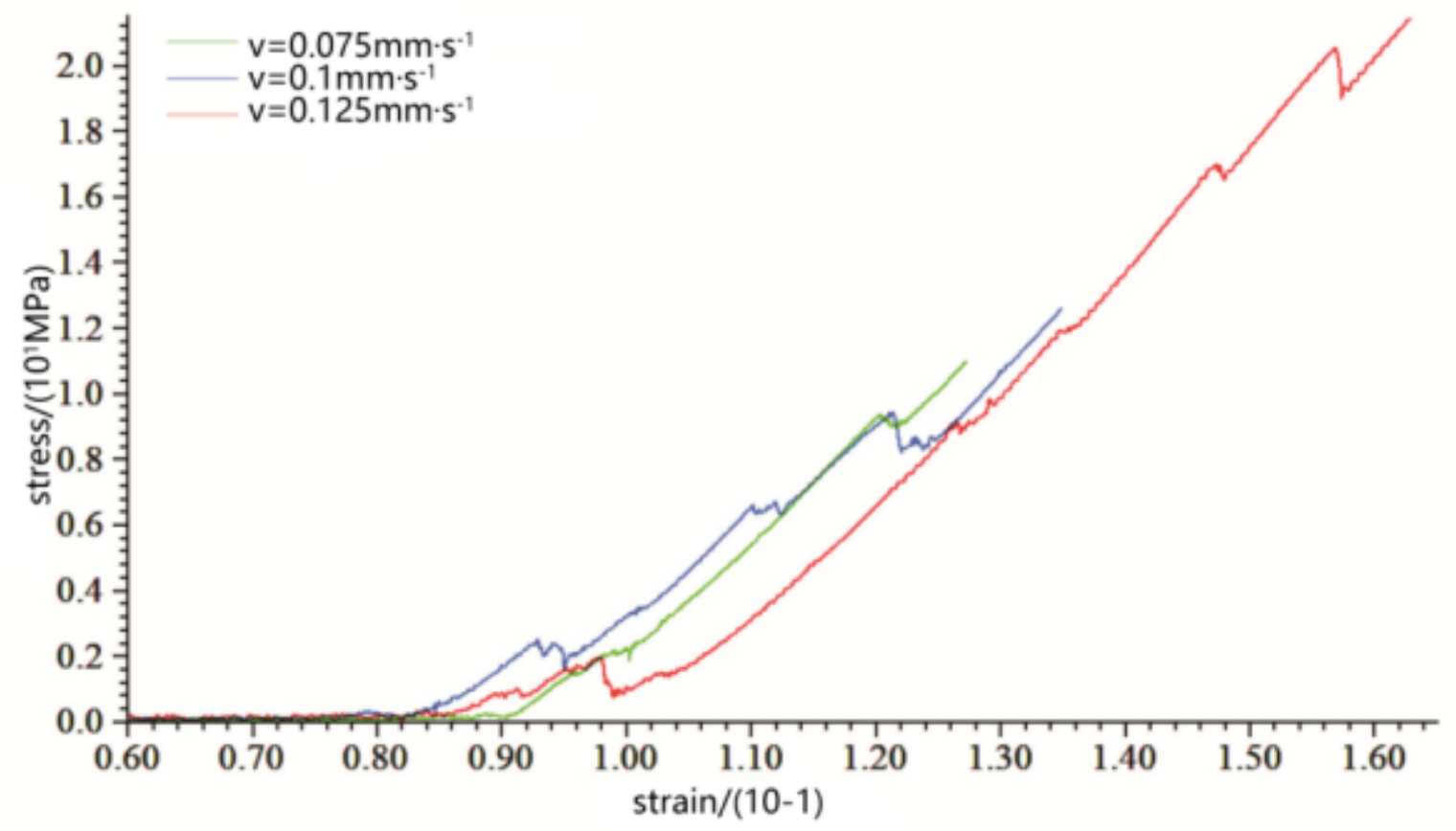

Figure 3

Stress-strain curves at different speeds 


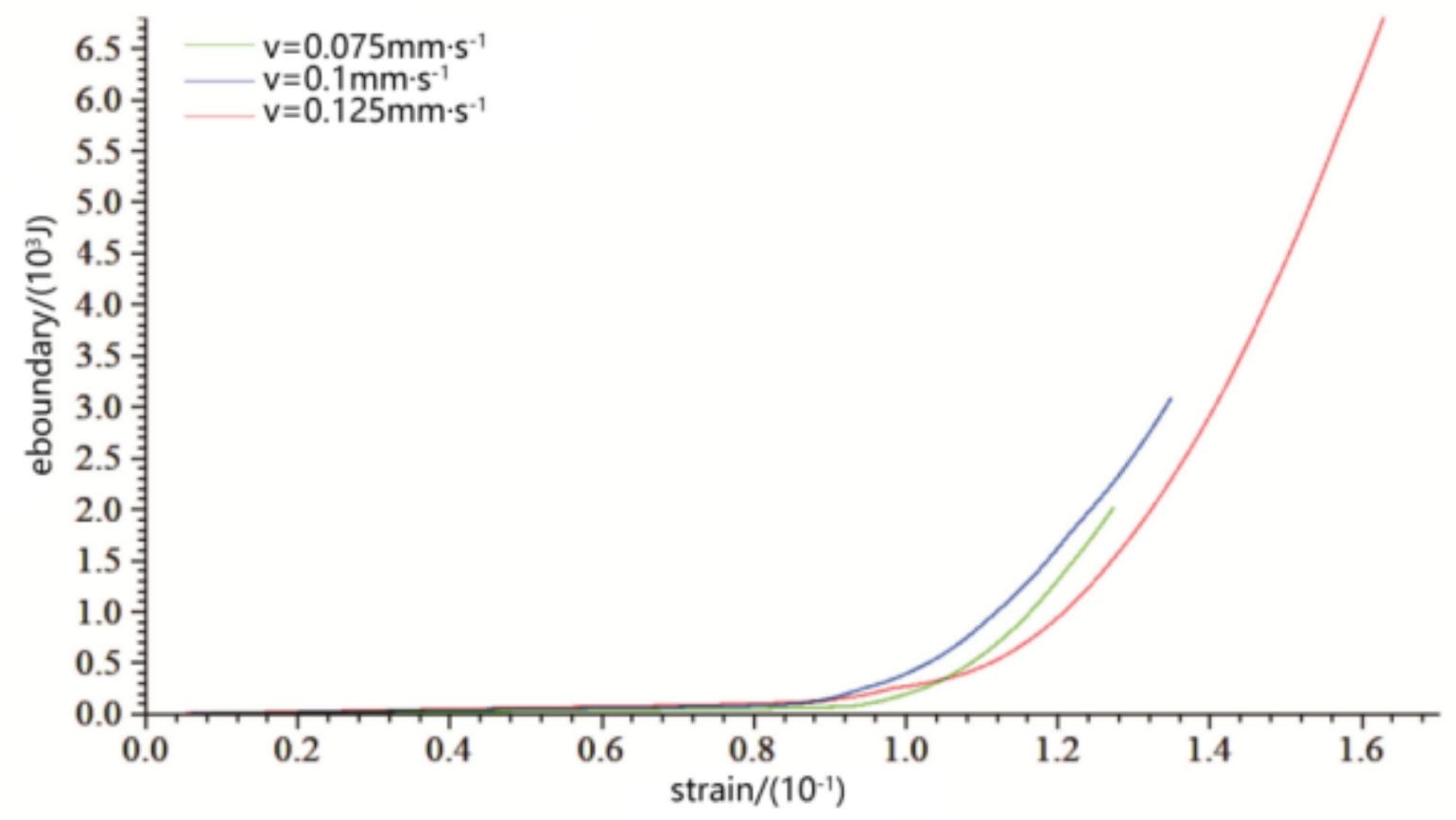

Figure 4

Boundary energy curves at different speeds

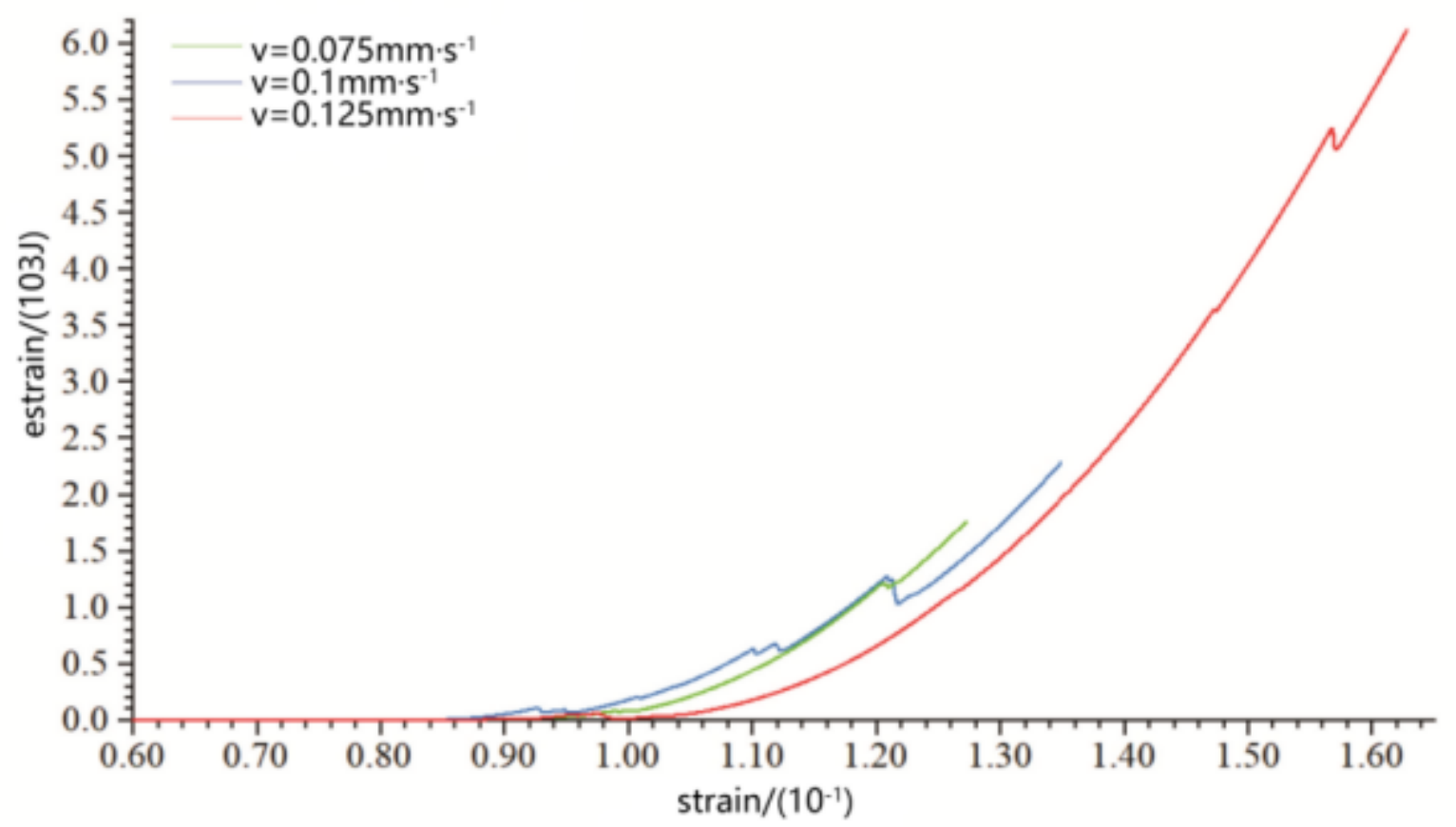

Figure 5

Cohesive energy curves at different speeds 


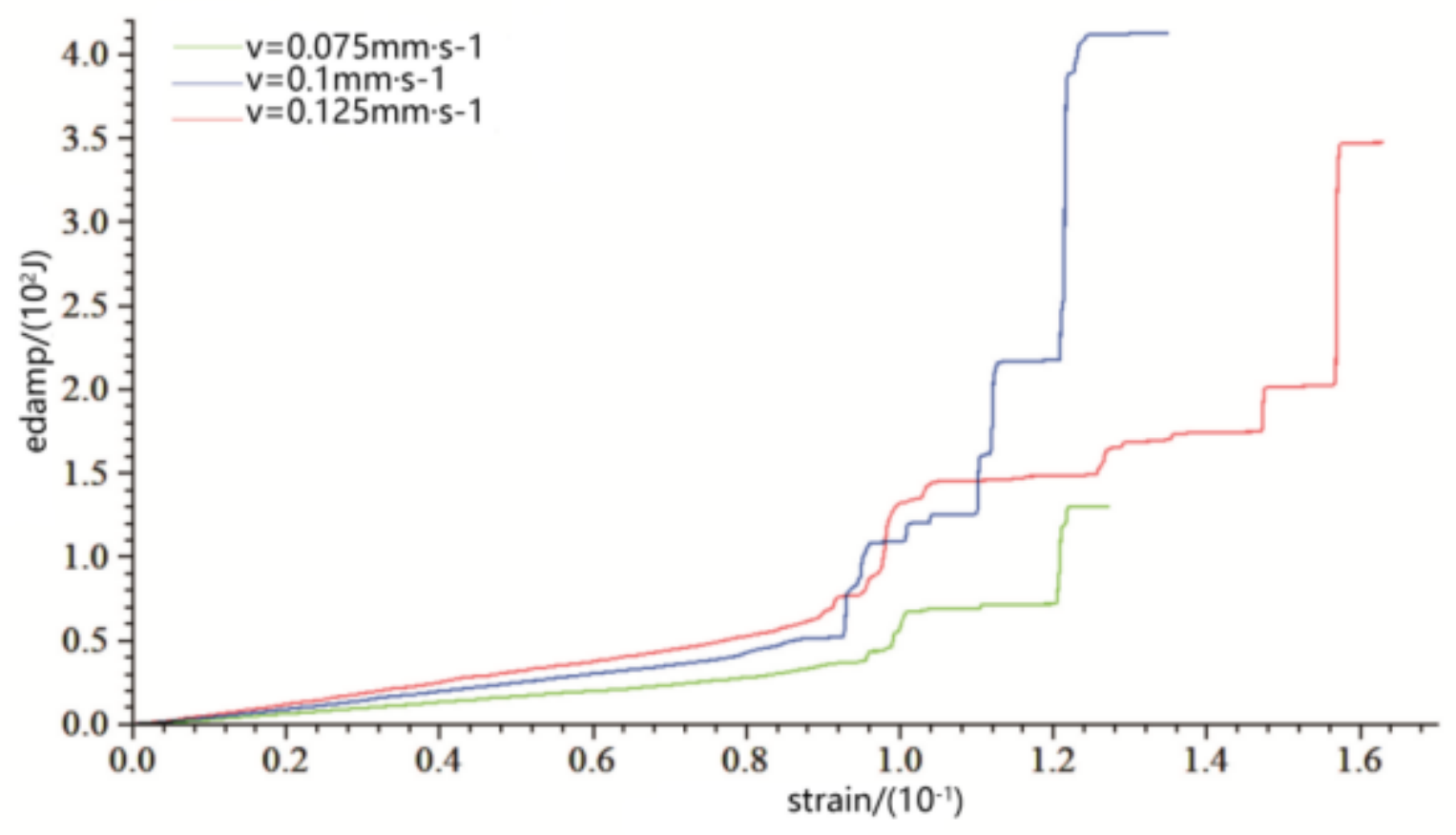

Figure 6

Friction energy curve at different speeds
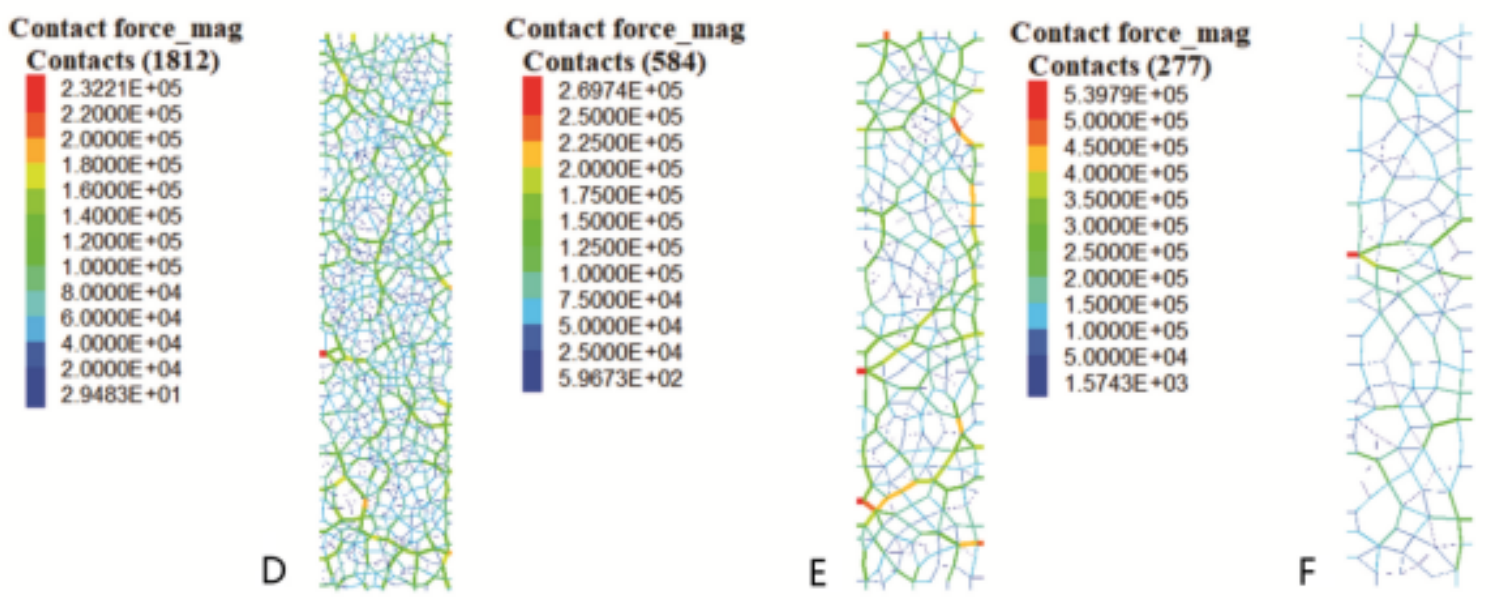

Figure 7

Force Chain Network under different granularities 


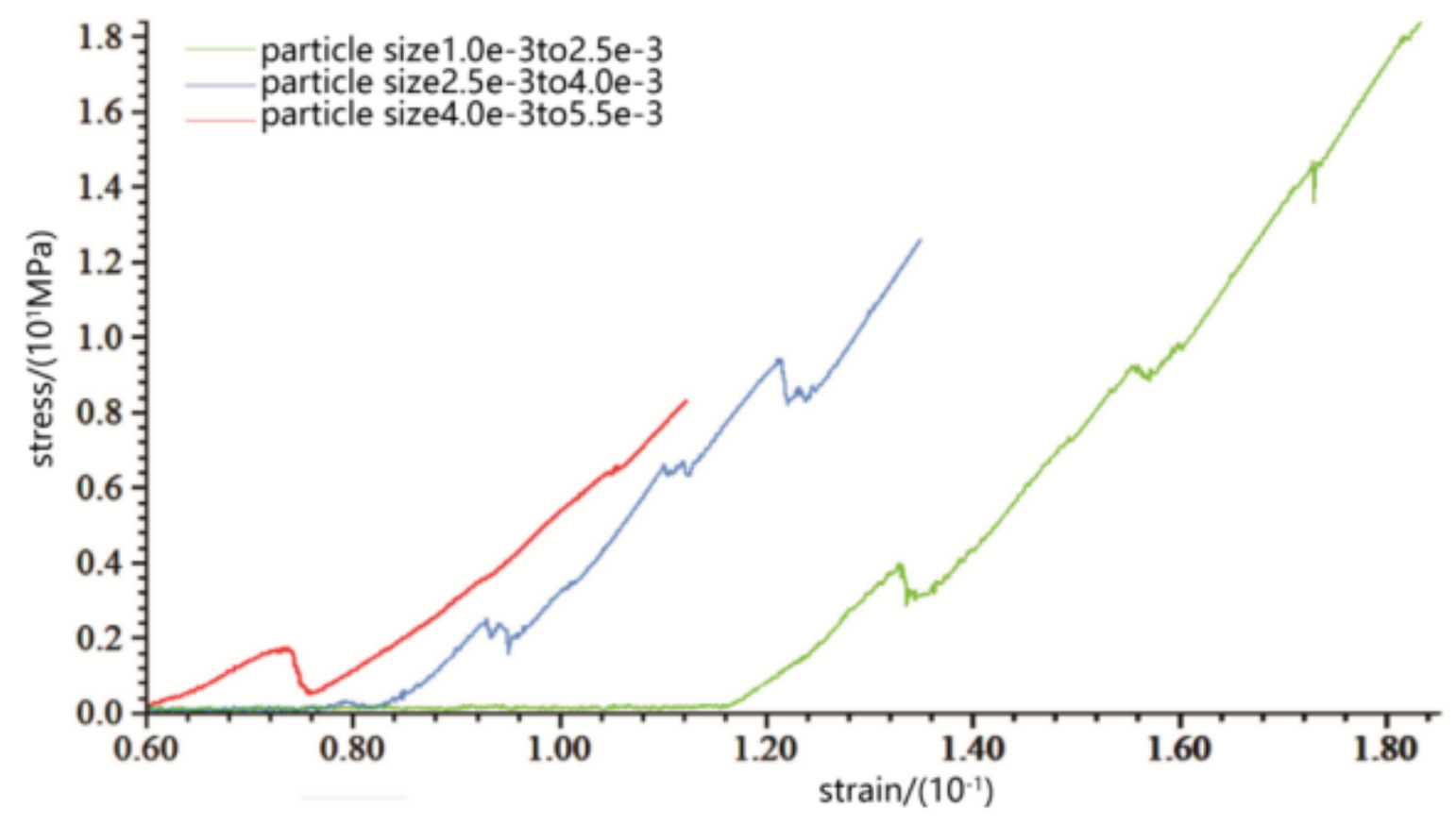

Figure 8

Stress-strain curves under different grain sizes

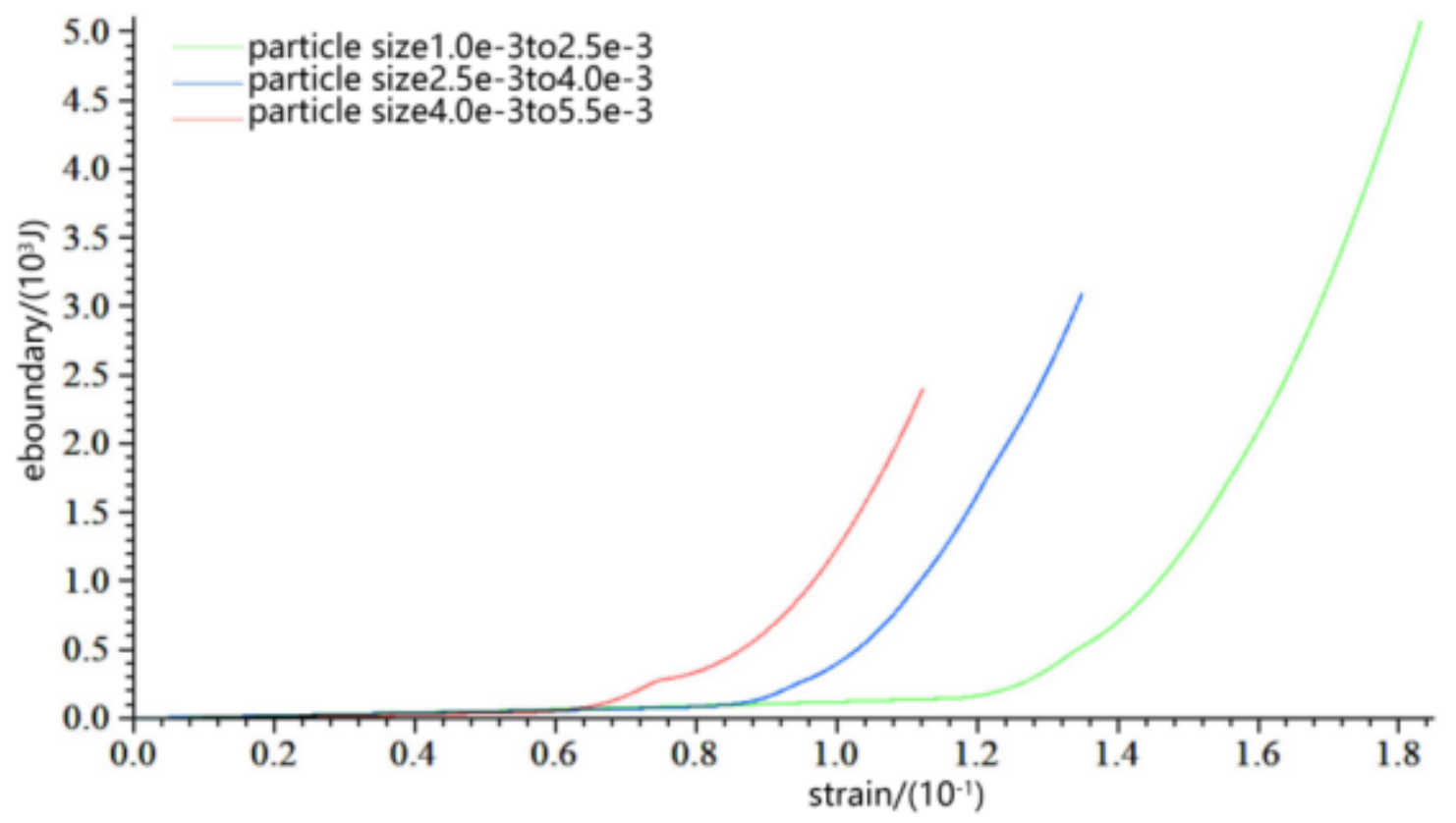

Figure 9

Boundary energy curves under different particle sizes 


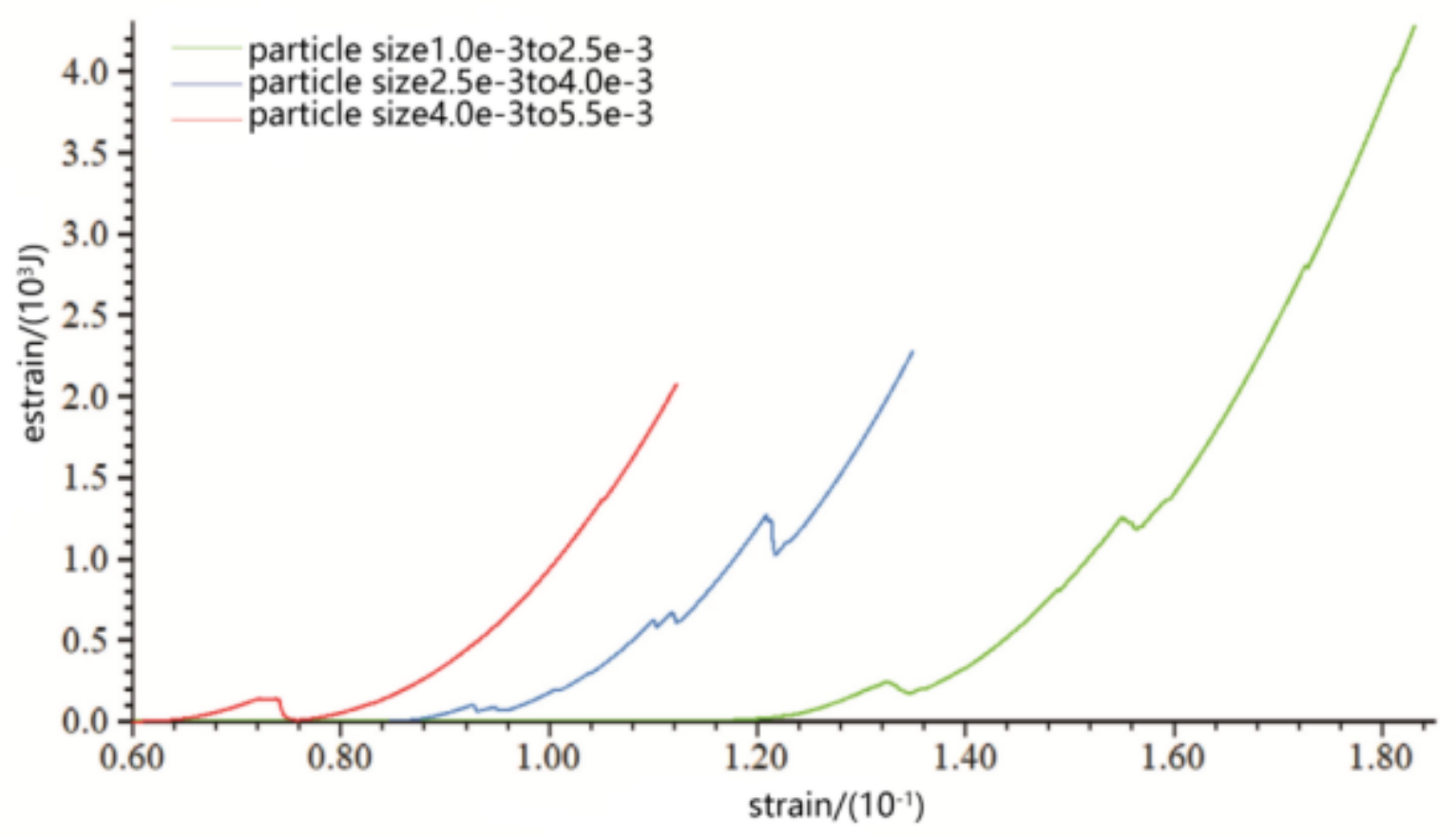

Figure 10

Cohesive energy curve under different particle sizes

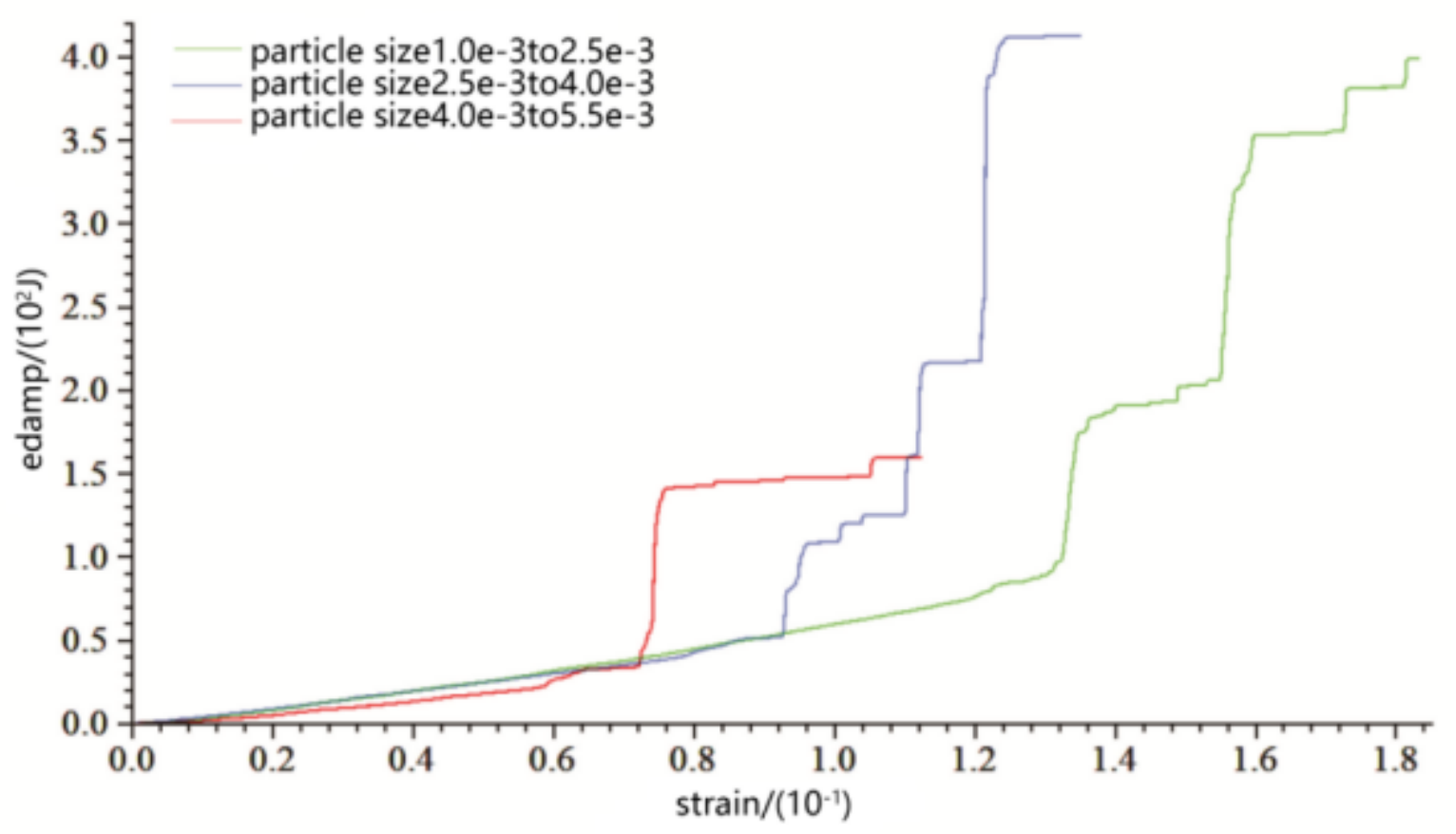

Figure 11

Friction energy curve under different particle sizes 